\title{
Microscopic activity in ulcerative colitis: what does it mean?
}

\author{
S A Riley, V Mani, M J Goodman, S Dutt, M E Herd
}

\begin{abstract}
To determine the prognostic importance of microscopic rectal inflammation we followed up 82 patients (aged 21 to 78 years, 44 men) with chronic quiescent ulcerative colitis over 12 months. At trial entry each patient underwent a rectal biopsy and sections were graded independently by two histopathologists. A chronic inflammatory cell infiltrate of varying severity was present in all biopsy specimens, and $58 \%$ had crypt architectural irregularities. In addition, $32 \%$ had evidence of acute inflammatory activity: $28 \%$ acute inflammatory cell infiltrate, $11 \%$ crypt abscesses, and $22 \%$ mucin depletion. Agreement between the two histopathologists for the presence of each of these features was $94 \%(90-98 \%)$. During the 12 month follow up 27 patients (33\%) relapsed after a mean interval of 18 weeks (range 3-44 weeks). Relapse rates were unrelated to duration or extent of disease or to the type of maintenance drug treatment. In patients with an acute inflammatory cell infiltrate $52 \%$ relapsed, whereas in the absence of such an infiltrate only $25 \%$ relapsed $(p=0 \cdot 02)$. Similarly, relapse rates were higher in the presence of crypt abscesses $(78 \% v 27 \%, \mathrm{p}<0.005)$, mucin depletion $(56 \% v \mathrm{p}<0.02)$, and breaches in the surface epithelium $(75 \% v 31 \%, p=0 \cdot 1)$. The presence of a chronic inflammatory cell infiltrate or crypt architectural irregularities, however, bore no relation to the frequency of colitis relapse.
\end{abstract}

The mucosal lesion in active ulcerative colitis is characterised by an intense inflammatory cell infiltrate, crypt abscesses, mucin depletion, and surface ulceration. During the healing phase epithelial continuity is restored, the infiltrate and abscesses begin to resolve, and epithelial mucin content improves. ${ }^{12}$ Histological recovery, however, is often incomplete and studies have shown that microscopic evidence of inflammation is common even in patients with clinically and sigmoidoscopically quiescent colitis. ${ }^{3-6}$

The prognostic importance of this microscopic inflammation is unknown. The aims of the present study were, therefore, to assess the relations between microscopic indices of inflammation and colitis relapse in a group of patients with quiescent disease.

\section{Methods}

\section{PATIENT SELECTION}

Adult outpatients with chronic ulcerative colitis in symptomatic and sigmoidoscopic remission were recruited to the study. Ulcerative colitis was diagnosed on the basis of clinical history and previous sigmoidoscopic and histological findings. Symptomatic remission was judged by a normal stool frequency and absence of blood in the stool for a minimum period of four weeks before inclusion.

The macroscopic appearances of the rectal mucosa were graded using well established criteria. ${ }^{7}$ Only patients with normal mucosal appearances or erythema were included in the study. Those with contact bleeding, spontaneous bleeding, or ulceration were specifically excluded as most of these patients have pronounced histological features of inflammation. ${ }^{5}$

Only patients taking either oral sulphasalazine or oral mesalazine as sole maintenance treatment were recruited. Those taking other drugs known to have an effect on colitis activity were excluded. No patient had received either oral or rectal steroids within four weeks of inclusion.

\section{HISTOLOGICAL ASSESSMENT}

At the time of sigmoidoscopy a mucosal biopsy specimen was taken from the anterior rectal wall between 5 and $10 \mathrm{~cm}$ from the anal margin. Biopsy specimens were fixed in formalin, embedded in paraffin, and 5 micron sections were stained with haematoxylin and eosin. Sections were then coded and graded independently by two histopathologists who had no knowledge of the patients.

Six histological features were assessed: the acute inflammatory cell infiltrate (polymorphonuclear cells in the lamina propria), crypt abscesses (Fig 1), mucin depletion (Fig 2), surface epithelial integrity, the chronic inflammatory cell infiltrate (round cells in the lamina propria), and crypt architectural irregularities. ${ }^{8}$ Each feature was graded on a four point scale corresponding to none, mild, moderate, or severe. The final grade being the mean of the two independent assessments.

\section{PATIENT FOLLOW UP}

Patients attended for clinical review at three, six, nine, and 12 months or at any other time if they wished. Throughout the study patients were asked to report promptly with symptomatic deterioration. Sigmoidoscopy was then undertaken and a stool culture performed. Colitis relapse was confirmed if the macroscopic appearance of the rectal mucosa had become haemorrhagic and the stool culture was negative.

All results are expressed as median and range unless otherwise stated. Relapse data were analysed using the $\chi^{2}$ test with Yates's correction. All other data were assessed using the MannWhitney U test. 


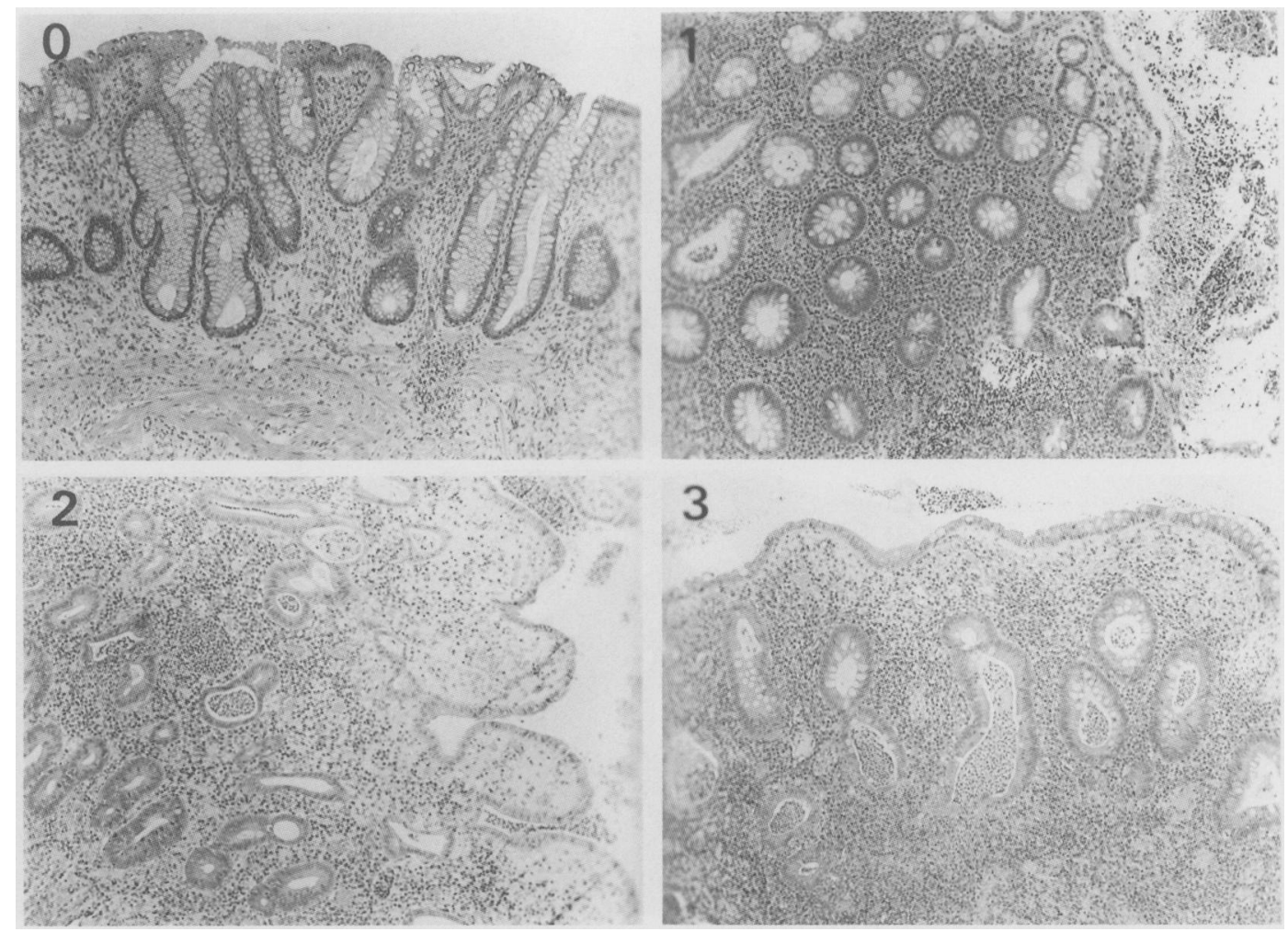

Figure 1: Crypt abscesses were graded on a four point scale corresponding to none, mild, moderate, or severe (0-3).

\section{Results}

\section{PATIENTS}

Eighty two patients were entered into the study, 44 men and 38 women, age range 21-78 years. The median disease duration was nine years (range $0 \cdot 5-34$ years). Disease extent, as judged by either double contrast barium enema or the macroscopic appearances at colonoscopy, was total in 17 patients and left sided in 18 , and showed proctosigmoiditis in 28 and proctitis in 19. All patients were maintained on either oral sulphasalazine ( 2 to $4 \mathrm{~g}$ daily) or oral mesalazine (800 to $1600 \mathrm{mg}$ daily) as sole treatment for the duration of the study.

\section{HISTOLOGICAL FEATURES}

Microscopic abnormalities were common in the 82 rectal biopsy specimens taken on entry to the study. All specimens showed a chronic inflammatory cell infiltrate and $48(58 \%)$ had crypt architectural irregularities. Four (5\%) showed breaches in the surface epithelium, $23(28 \%)$ had an acute inflammatory cell infiltrate, nine (11\%) had crypt abscesses, and 18 (22\%) had mucin depletion.

The degree of observer variation for each of the histological features is shown in Figure 3. Agreement between the two histopathologists for the presence or absence of each of the six features was $94 \%(90-98 \%)$. Furthermore, the same grade was allocated to each histological feature in $82 \%(50-98 \%)$ of the biopsy sections and differed by more than one grade in only $2 \%$ $(0-6 \%)$ of the sections. Observer variability was less when grading crypt abscesses, mucin depletion, and epithelial integrity than when grading either the degree of acute or chronic cellular infiltration or the degree of crypt architectural irregularities.

Twenty six of the 82 biopsy specimens (32\%) had features indicative of acute inflammation. As might be expected, combinations of the acute inflammatory indices within a biopsy specimen were common (Fig 4) such that all patients who had crypt abscesses had an acute inflammatory cell infiltrate and most also had mucin depletion.

The presence of acute inflammatory activity in relation to the time from previous colitis relapse before inclusion in the study is shown in Figure 5. Patients with indices of acute activity had suffered a more recent relapse (median 5 (range 2-37) months) than those who had not (9 (1-72) months), but this fell just short of significance $(p=0 \cdot 06)$.

\section{CLINICAL OUTCOME}

During the 12 month follow up 27 patients (33\%) relapsed after a median interval of 18 weeks (range 3-44 weeks). Relapse rates were unrelated to age, sex, disease duration, disease extent, or type of maintenance drug treatment. Furthermore, the frequency of colitis relapse was unrelated to the macroscopic appearances of the

Severity of the inflammatory cell infiltrate in relation to relapse rate

\begin{tabular}{|c|c|c|c|}
\hline $\begin{array}{l}\text { Acute inflammatory cell infiltrate: } \\
\text { Histological grade } 0 \text { or } 0.5 \\
\text { No of patients per group } 67 \\
\text { No of patients relapsing }(\%) 17(25)\end{array}$ & $\begin{array}{l}1 \text { or } 1 \cdot 5 \\
13 \\
8(61)^{\star}\end{array}$ & $\begin{array}{l}2 \text { or } 2 \cdot 5 \\
2 \\
2(100)\end{array}$ & $\begin{array}{l}3 \\
0 \\
-\end{array}$ \\
\hline $\begin{array}{ll}\text { Chronic inflammatory cell infiltrate: } \\
\text { Histological grade } & 0 \text { or } 0.5 \\
\text { No of patients per group } & 5 \\
\text { No of patients relapsing }(\%) & 2(40)\end{array}$ & $\begin{array}{l}13 \text { or } 1 \cdot 5 \\
53 \\
16(30)\end{array}$ & $\begin{array}{l}2 \text { or } 2 \cdot 5 \\
20 \\
7(35)\end{array}$ & $\begin{array}{l}3 \\
4 \\
2(5)\end{array}$ \\
\hline
\end{tabular}

${ }^{\star} \mathrm{p}<0.02$ compared to grade 0 or 0.5 group. 


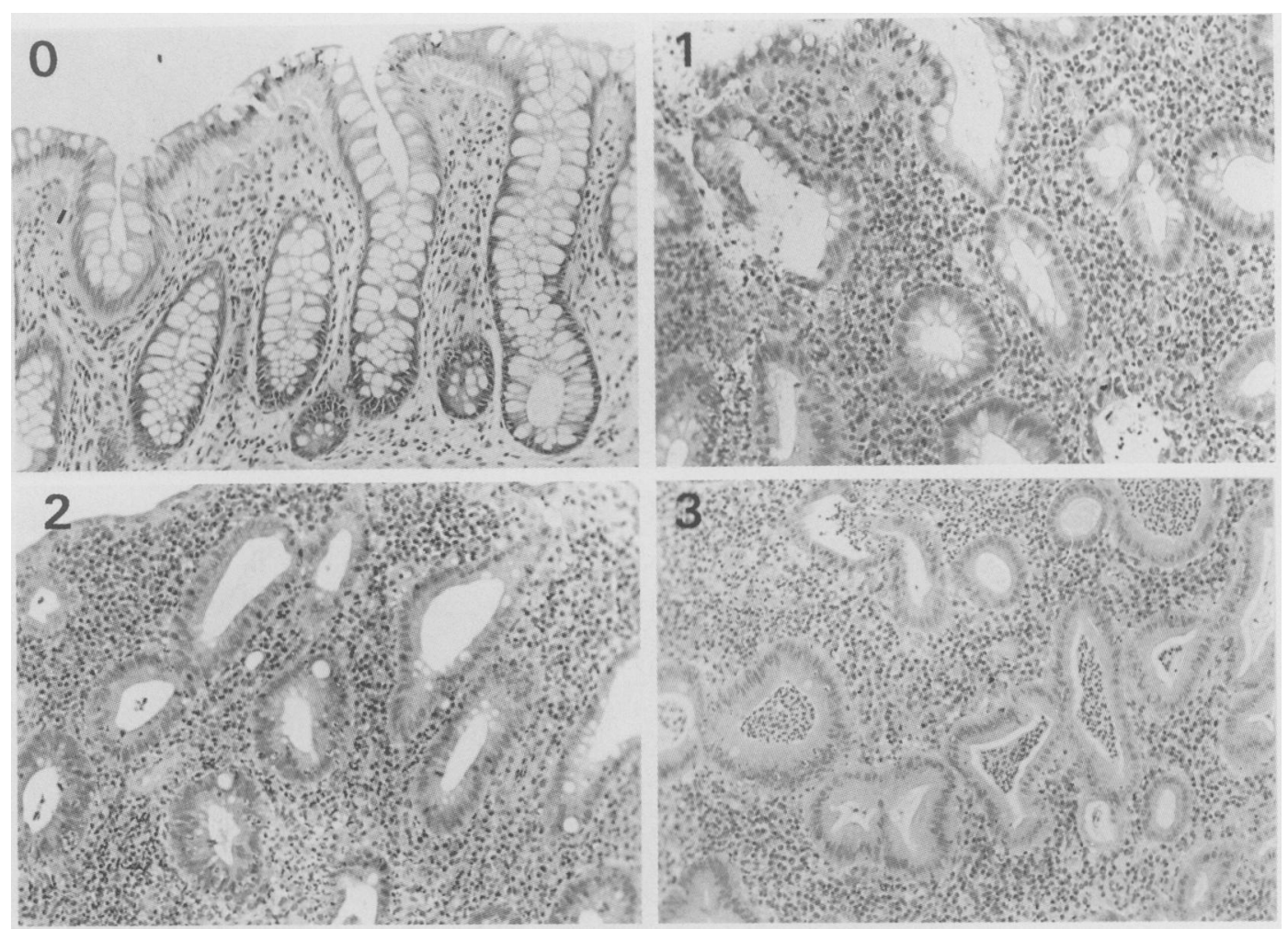

Figure 2: Mucin depletion was graded on a four point scale corresponding to none, mild, moderate, or severe (0-3).

rectal mucosa at the time of trial entry. Relapse occurred in $32 \%$ of patients with mucosal erythema and in $35 \%$ of those with completely normal sigmoidoscopic appearances.

The influence of histological features on the frequency of colitis relapse is shown in Figure 6. In patients whose rectal biopsy specimens showed an acute inflammatory cell infiltrate the relapse rate was $52 \%$, whereas in the absence of such an infiltrate only $25 \%$ of the patients relapsed $(p=0.02)$. Similarly, relapse rates were considerably higher in the presence than in the absence of crypt abscesses $(78 \% \quad v \quad 27 \%$, $\mathrm{p}<0.005)$, mucin depletion $(56 \%$ v $26 \%$, $\mathrm{p}<0.02$ ), and breaches in the surface epithelium

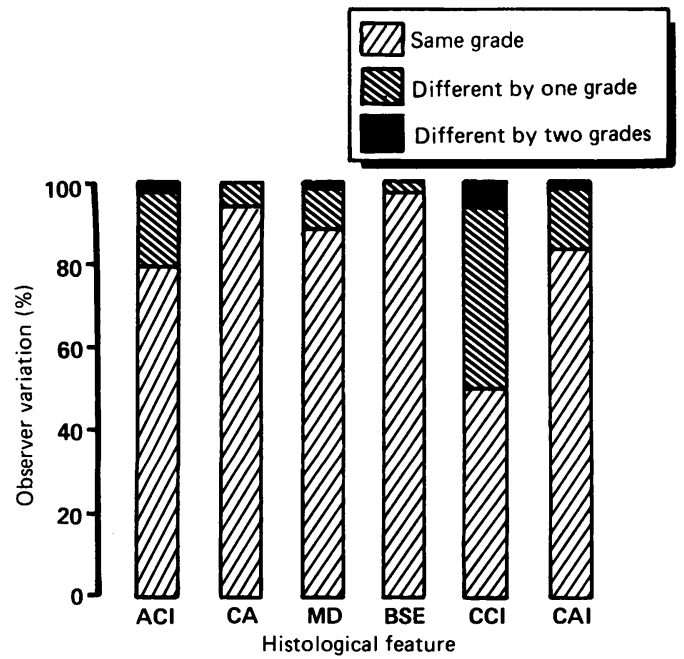

Figure 3: The degree of observer variation for each histological feature. All features were graded on a four point scale by two histopathologists. ACI=acute inflammatory cell infiltrate, $C A=$ crypt abscesses, $M D=$ mucin depletion, $B S E=$ breached surface epithelium, $C C I=$ chronic inflammatory cell infiltrate, $C A I=$ crypt architectural irregularities.
$(75 \% v 31 \%, \mathrm{p}=0 \cdot 1)$. The presence of a chronic inflammatory cell infiltrate or crypt architectural irregularities had no clear effect on subsequent relapse rate.

The Table shows the relation between the severity of the inflammatory infiltrate and the frequency of colitis relapse. Of the patients with an acute inflammatory cell infiltrate graded 0 or 0.5 , only $25 \%$ relapsed. Among those with an acute infiltrate graded 1 or 1.5 , however, $61 \%$ relapsed $(\mathrm{p}<0 \cdot 02)$. Only two patients had biopsy specimens graded at a higher level and both relapsed. No relation was apparent between the severity of the chronic inflammatory cell infiltrate and subsequent relapse rate.

Of the patients who relapsed, those with histological evidence of acute inflammatory activity on entry to the study tended to relapse after a shorter time interval than those without. This difference, however, was not significant (median 12 (range 4-44) weeks v 16 (3-40) weeks).

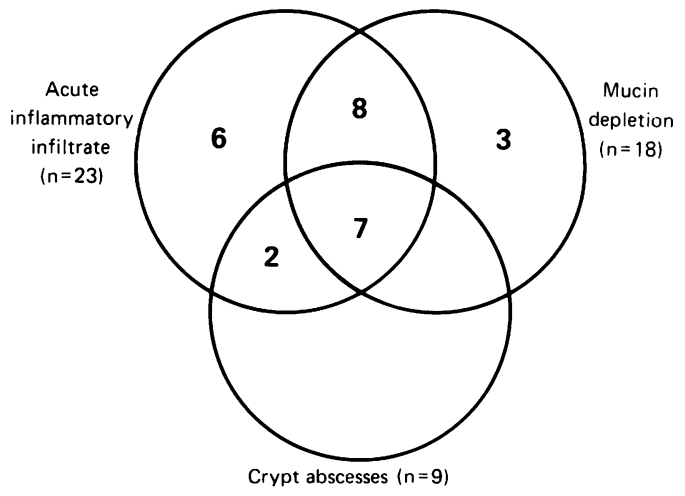

Figure 4: The combinations of acute inflammatory indicators in biopsy sections. 


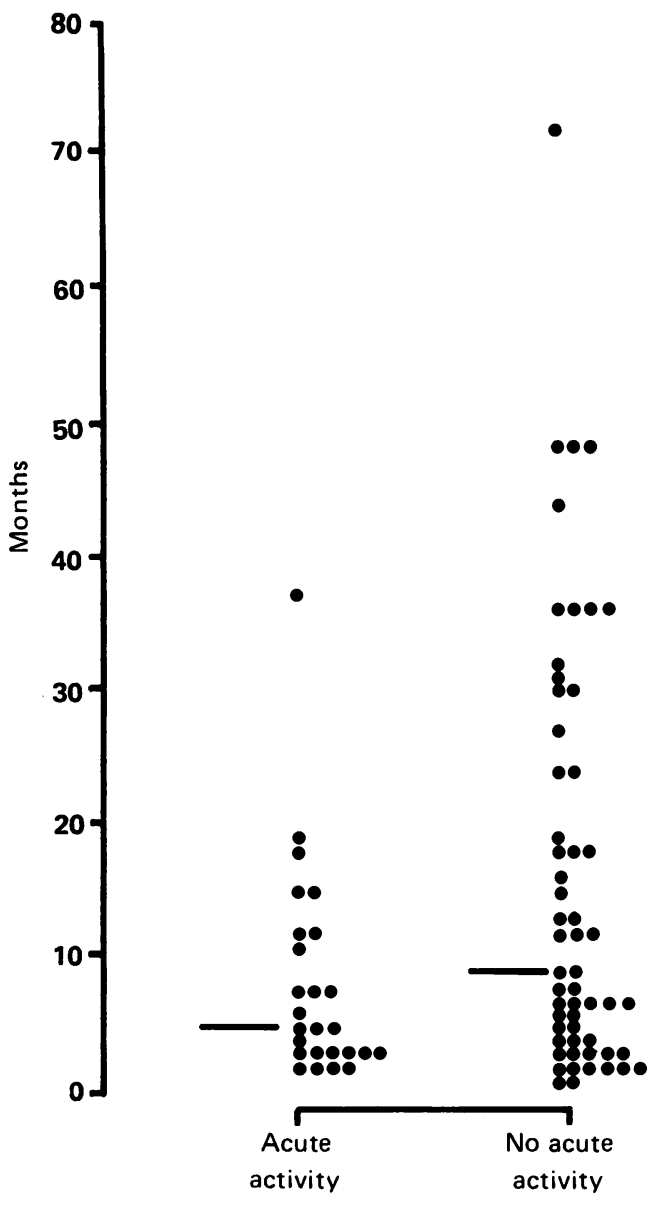

Figure 5: The presence of acute inflammatory activity in relation to the time from previous colitis relapse before inclusion in the study (median 5 (range 2-37) months v 9 (172) months, $p=0.06$ ).

\section{Discussion}

It is well recognised that most patients with ulcerative colitis run a relapsing course. When symptoms improve after treatment for an acute attack, however, macroscopic evidence of the inflammatory process on sigmoidoscopy often lingers behind. ${ }^{9}$ Moreover, histological indicators of inflammaton are common even in patients who achieve completely normal mucosal appearances.

In one of the earliest studies Truelove and Richards found that $60 \%$ of patients in clinical remission had abnormal sigmoidoscopic features

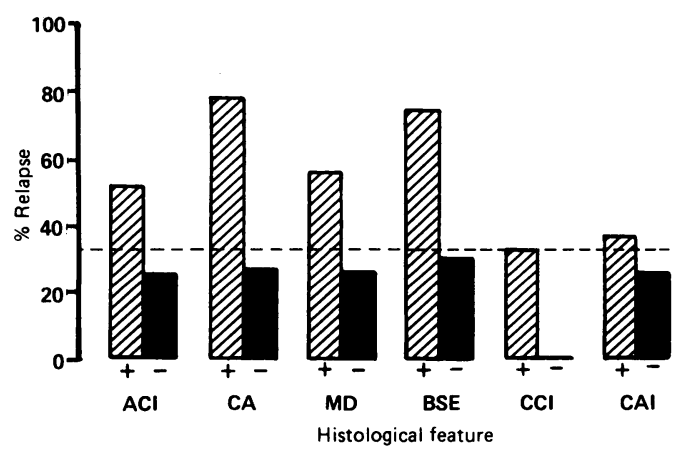

Figure 6: The influence of histological features on the frequency of colitis relapse. The dotted line indicates the relapse rate for the group as a whole $(33 \%) . A C I=$ acute inflammatory cell infiltrate $(p=0.02), C A=$ crypt abscesses $(p<0.005), M D=$ mucin depletion $(p<0.02), B S E=$ breached surface epithelium, $C C I=$ chronic inflammatory cell infiltrate, $C A I=$ crypt architectural irregularities. and $37 \%$ of patients with normal macroscopic appearances had histological evidence of mild or moderate inflammation. ${ }^{3}$ Matts found microscopic inflammation in 15 of 23 patients (65\%) with clinically and sigmoidoscopically quiescent disease $^{4}$ and similar results have been reported by others. ${ }^{5} 1011$

In a study attempting to relate microscopic features to the clinical course of colitis, Dick et al found abnormal histology in 43 of 48 patients $(90 \%)$ with normal mucosal appearances. ${ }^{12}$ There seemed to be no relation between the histological features and time from previous colitis relapse, but unfortunately controlled clinical follow up was not undertaken. Furthermore, the microscopic findings in this study were unusual because acute inflammatory indicators were never noted in patients with a macroscopically normal rectal mucosa. This is in striking contrast to the findings of Sommers and Korelitz who reported an almost tenfold increase in neutrophil count in biopsy specimens from patients with normal sigmoidoscopic appearances compared with control subjects. ${ }^{13}$

The present study confirms the high prevalence of histological abnormalities in patients with clinically and sigmoidoscopically quiescent colitis. A chronic inflammatory cell infiltrate was present in all specimens and over half had crypt architectural abnormalities. Neither of these features, however, were of prognostic importance. Acute inflammatory indicators, on the other hand, present in $32 \%$, were associated with a two to threefold increased risk of colitis relapse during 12 month follow up.

In a controlled trial of various dietary treatments for ulcerative colitis Wright and Truelove ${ }^{14}$ undertook serial rectal biopsies in a group of 77 patients over a one year period. They too reported a twofold increase in relapse rate in patients with appreciable microscopic inflammation. Furthermore, histological features seemed to be of greater predictive value than the macroscopic appearances of the rectal mucosa.

The reason why almost one third of patients had microscopic evidence of acute inflammation is obscure. One possibility is that these findings represent residual damage from a recent previous relapse. This seems unlikely, however, as the association between time from previous colitis relapse and acute inflammatory activity fell short of statistical significance and overlap between the two groups was considerable. It is more likely that these patients represent a subgroup of ulcerative colitis at increased risk of relapse. Poor drug compliance may be a factor, and this is a well recognised problem in patients taking maintenance sulphasalazine treatment. ${ }^{15}$ We feel, however, that poor compliance is not the sole explanation for these findings as we routinely assess and encourage compliance at clinic attendance and frequent relapses may, in themselves, prompt good compliance.

It was encouraging to find that observer reproducibility was good despite the use of a simple subjective grading system. Reproducibility was particularly good when crypt abscesses and mucin depletion were assessed, both of which were useful prognostic indicators. We therefore suggest that routine histological 
assessment of these features may be used as simple prognostic indicators without recourse to detailed morphometric analysis.

Two practical issues arise from this study. Firstly, we have identified a subgroup of patients who seem to be at particular risk of colitis relapse. Therapeutic trials of either high dose maintenance sulphasalazine or mesalazine or perhaps short term treatment with topical corticosteroids therefore seem to be indicated. Secondly, the results of this study suggest that future maintenance drug trials in ulcerative colitis should stratify patients according to the presence of microscopic acute inflammation. The inclusion of such patients may explain why recent trials report higher relapse rates ${ }^{16}{ }^{17}$ than those reported in earlier studies of patients in prolonged clinical and histological remission. ${ }^{18} 19$

We are most grateful to Professor L A Turnberg for allowing us to study patients under his care and his helpful comments on the manuscript.

1 Morson BC. Rectal biopsy in inflammatory bowel disease. NEngl F Med 1972; 287: 1337-9.

2 Flick AL, Voegtlin KF, Rubin CE. Clinical experience with suction biopsy of the rectal mucosa. Gastroenterology 1972; 42: 691-705.

3 Truelove SC, Richards WCD. Biopsy studies in ulcerative colitis. BrMed F 1956; i: 1315-8.

4 Matts SGF. The value of rectal biopsy in the diagnosis of ulcerative colitis. Of Med 1961; 30: 393-407.

5 Powell-Truck J, Day DW, Buckell NA, Wadsworth J, Lennard-Jones JE. Correlations between defined sig- moidoscopic appearances and other measures of disease activity in ulcerative colitis. Dig Dis Sci 1982; 27: 533-7.

6 Korelitz BI, Sommers SC. Responses to drug therapy in ulcerative colitis. Am $\mathcal{F}$ Gastroenterol 1975; 64: 365-70.

7 Baron JH, Conell AM, Lennard-Jones JE. Variation between observers in describing mucosal appearances in proctoobservers in describing mucosal appearances in proctocolitis. BrMed f 1964; 1: 89-92.

8 Morson BC, Dawson IMP, In: Gastrointestinal pathology. Oxford: Blackwell Scientific, 1972: 458-75.

9 Fochios DE, Korelitz BI. The role of sigmoidoscopy and rectal biopsy in diagnosis and management of inflammatory bowe disease: personal experience. Am $\mathcal{f}$ Gastroenterology 1988 83: 114-9.

10 Watts JM, Thompson H, Goligher JC. Sigmoidoscopy and cytology in the detection of microscopic disease of the rectal mucosa in ulcerative colitis. Gut 1966; 7: 288-94.

11 Binder V. A comparison between clinical state, macroscopic and microscopic appearance of rectal and cytologic picture of mucosal exudate in ulcerative colitis. Scand $\mathcal{F}$ Gastroenterol mucosal exudate

12 Dick AP, Holt LP, Dalton ER. Persistence of mucosal abnormality in ulcerative colitis. Gut 1966; 7: 355-60.

13 Sommers SC, Korelitz BI. Mucosal cell counts in ulcerative and granulomatous colitis. Am $\mathcal{F}$ Clin Pathol 1975; 63: $359-65$.

14 Wright $R$, Truelove SC. Serial rectal biopsy in ulcerative colitis during the course of a controlled therapeutic trial of various diets. Am $\mathcal{F}$ Dig Dis 1966; 11: 847-57.

15 Van Hees PAM, Van Tongeren JHM. Compliance to therapy in patients on a maintenance dose of sulphasalazine. $7 \mathrm{Clin}$ Gastroenterol 1982; 4: 333-6.

16 McIntyre PB, Rodrigues CA, Lennard-Jones JE, et al. Balsalazide in the maintenance treatment of patients with ulcerative colitis, a double-blind comparison with sulphasalazine. Aliment Pharmacol Therap 1988; 2: 237-43.

17 Mulder CJJ, Tytgat GNJ, Weterman IT, et al. Double-blind comparison of slow-release 5-aminosalicylate and sulphasalazine in remission maintenance in ulcerative colitis. Gastroenterology 1988; 95: 1449-53.

18 Dissanayake AS, Truelove SC. A controlled therapeutic trial of long-term maintenance treatment of ulcerative colitis with sulphasalazine (Salazapyrin). Gut 1973; 14: 923-6.

19 Azad Khan AK, Howes DT, Piris J, Truelove SC. Optimum dose of sulphasalazine for maintenance treatment of ulcerative colitis. Gut 1980; 21 : 232-40. 